\title{
References.
}

(1) A. Szent-György: Biochem. J., 22, 1387, (1928).

( 2 ) J. Tillmans, P. Tirsch and R. Vaubel : Z. Unters. Lebensmitt,, 60, 34, (1930); 63, (1952), 1, 21, 241, 267, 276; 64, 11, (1932); 65, 145, (1933).-Biochem. Z., 250, 312, (1932).

(3) A. Szent-Györgyi and J. L. Svirbely: Nature, 129, 576, 690, (1932).

(4) W. A. Waugh and C. G. King: Science, 75, 1944, (1932).

(5) J. Tillmans, P. Hirsch and R. Vaubel: Z. Unters. Lebensmitt., 65, 145, (1933); J. Brüggemann: Z. f. Physiol, Chem,, 216, 139, (1933).

(6) Svirbely and Szent-Györgyi: Biochem. J., 27, 279, (1933).

(7) V. Demole: Z, f. Physiol. Chem., 217, 83, (1933).

(8) F. Micheel and Th. Moll: Z. f, Physiol. Chem., 219, 253, (1933).

(9) T. Reichstein, A. Grïssner and R. Oppenauer: Helv. Chem. Acta, 16, 561, 1020, (1933) ; 17, 312. (1934).

(10) M. Tsujimura: Bull. I. P. C. R., 5, 701, (1926). (written in Japanese)

(11) M. Miura and M. Tsujimura: Sc. Pap. I. P. C. R., 20, (1933), No. 409, 129. (Translated into English from the Journal of the Agricultural Chemical Society of Japan. Vol. I, No. 1. 1924) ; M. Miura and N. Okabe: Sc. Pap. I. P. C. R., 20, No. 410, 145, (1933). (Translated into English from their original paper written in Japanese, 1926).

\section{Kationenumtausch an den Tonen. (I. Mitteilung)}

\author{
Von \\ Matuo TokuokA. \\ (Agrikulturchemische Abteilung, Taihoku kaiserliche Universität, Taihoku, Taiwan, Japan.)
}

(Eingegagen am 12, September 1934)

Zur Aufklärung der Beziehung von Kationenumtausch und Struktur bei den Tonen, hat der Verfasser vier Sorten der Tonen, d. h., Bentonit, Hettenleidelheimer Fettton, Saarauer Blauton und Zettlitzer Kaolin, deren Eigenschaften von einander sehr verschieden sind, als die Versuchsmaterialien gebraucht. Sie wurden zu erst als Calciumton geändert. Dann wurden die ausgetauschte $\mathrm{Ca}-\mathrm{Menge}$ gegen verschiedene Aminen mit verschiedenen Ionengrössen bestimmt. Die Ionengrösse der einzutauschender Kationen haben auf die ausgetauschte Menge des Calciums keine Einflüsse ausgeübt. Daraus hat der Verfasser so zusammengefasst, dass der Kationenumtausch an den Tonen nur auf der Oberfläche stattfinden soll.

Die Werte, die nach der Freundlichschen Formel berechnet wurden, haben mit den experimentellen werten sehr gut übereinstimmt. 